\title{
Simulations of $S U(2)$ lattice gauge theory with dynamical reduced staggered fermions
}

\author{
Simon Catterall ${ }^{*}$ and Nouman Butt ${ }^{\dagger}$ \\ Department of Physics, Syracuse University, Syracuse, New York 13244, USA
}

(Received 21 November 2018; published 14 January 2019)

\begin{abstract}
We simulate $S U(2)$ lattice gauge theory in four dimensions using dynamical reduced staggered fermions. The latter lead to two rather than four Dirac fermions in the continuum limit. We review the derivation and properties of reduced staggered fermions and show that in the case of fields in the fundamental representation of $S U(2)$ the theory does not exhibit a sign problem and can be simulated using the rational hybrid monte carlo algorithm. We present results on lattices up to $16^{4}$ for a wide range of bare fermion masses. We find a single site condensate appears at strong coupling that spontaneously breaks the one global $U(1)$ symmetry remaining in the reduced fermion action.
\end{abstract}

DOI: 10.1103/PhysRevD.99.014505

\section{INTRODUCTION}

Simulations of gauge theories using staggered fermions have a long history going back to the early days of lattice gauge theory. In recent years they have allowed for precision studies of hadronic quantities of crucial importance to experimental efforts to test and constrain the Standard Model [1].

As is well known the four dimensional naive staggered fermion action yields not one but four Dirac fermions in the continuum limit. It is less well appreciated that this replication can be halved by an additional thinning of lattice degrees of freedom to create what are called reduced staggered fermions. At first glance this fact seems to imply that the reduced fermion would be a better choice than the usual staggered fermion for simulations. It was realized early on that this was not the case; for QCD the resulting fermion determinant is not real, positive definite, and furthermore it is not possible to write gauge invariant single site mass terms in such a theory [2].

In this paper we point out that these problems can be evaded for gauge groups with pseudoreal representations. As an example we consider the case of fermions transforming in the fundamental representation of $S U(2)$. Quenched simulations of this model have been studied in [3] but the only work we are aware of with dynamical fermions was carried out in the context of a four fermion

\footnotetext{
*smcatter@syr.edu

†ntbutt@syr.edu
}

Published by the American Physical Society under the terms of the Creative Commons Attribution 4.0 International license. Further distribution of this work must maintain attribution to the author(s) and the published article's title, journal citation, and DOI. Funded by SCOAP. model with off site Yukawa couplings [4]. In this paper we study the case of the $S U(2)$ gauge theory with both single site and one-link mass terms. We show that the corresponding single site fermion condensate dominates at strong coupling in the thermodynamic limit as the fermion masses are sent to zero. The appearance of a single site condensate breaks the one remaining global $U(1)$ symmetry in the reduced fermion action and leads to a light pion which is also measured in our simulations. This $U(1)$ symmetry breaking is consistent with the random matrix theory analysis of [5].

One of our motivations for this work comes from recent studies of a four fermion model built from four reduced staggered fermions. In three dimensions there is good evidence that the weak and strong couplings phases of this theory are separated by a continuous phase transition with nontrivial critical exponents [6,7]. Moreover, while no symmetries are broken in the strong coupling phase, the system nevertheless generates a mass gap. Thus the phase transition does not seem to be describable in terms of a Landau-Ginzburg effective theory [8].

In four dimensions a very narrow broken symmetry phase reappears between the weak and strong coupling phases $[9,10]$, but there is evidence that this broken symmetry phase may be evaded in an expanded phase diagram corresponding to a Higgs-Yukawa generalization of the model $[11,12]$. This latter model possesses a global $S O(4)=S U(2) \times S U(2)$ symmetry with the Yukawa interaction coupling the staggered fermions to a scalar field living in the adjoint representation of one of these $S U(2)$ s. A natural extension of this model then replaces the scalar field with an $S U(2)$ gauge field which we conjecture is capable of generating the same four fermion condensate now via strong gauge interactions. As a first step in this direction we need to understand the phase structure and symmetry breaking patterns of reduced 
staggered fermions interacting via a $S U(2)$ gauge fieldthe study reported here.

\section{ACTION AND SYMMETRIES}

For completeness we repeat here the derivation of the reduced staggered fermion action [2]. Starting with the full massless staggered action

$$
\begin{aligned}
S_{F}= & \sum_{x, \mu} \frac{1}{2} \eta_{\mu}(x) \bar{\psi}(x)\left[U_{\mu}(x) \psi(x+\mu)\right. \\
& \left.-U_{\mu}^{\dagger}(x-\mu) \psi(x-\mu)\right],
\end{aligned}
$$

where the staggered fermion phases are given by

$$
\eta_{\mu}(x)=(-1)^{\sum_{i=1}^{\mu-1} x_{i}},
$$

we project down to reduced staggered variables.

$$
\begin{aligned}
& \bar{\psi}(x) \rightarrow \frac{1+\epsilon(x)}{2} \bar{\psi}(x) \\
& \psi(x) \rightarrow \frac{1-\epsilon(x)}{2} \psi(x),
\end{aligned}
$$

where the parity factor $\epsilon(x)=(-1)^{\sum_{i=1}^{4} x_{i}}$. Since $\bar{\psi}$ is only defined on even sites we can relabel it as $\psi^{T}$. Furthermore, we can introduce a new gauge field $\mathcal{U}_{\mu}(x)$ defined by

$$
\mathcal{U}_{\mu}(x)=\frac{1+\epsilon(x)}{2} U_{\mu}(x)+\frac{1-\epsilon(x)}{2} U_{\mu}^{*}(x)
$$

and rewrite the resultant reduced staggered action in the form

$$
S_{F}=\sum_{x, \mu} \frac{1}{2} \eta_{\mu}(x) \psi^{T}(x) \mathcal{U}_{\mu}(x) \psi(x+\mu) .
$$

By taking the transpose of this equation it can be written equivalently as

$$
S_{F}=\sum_{x, \mu} \frac{1}{2} \psi^{T}(x) \eta_{\mu}(x) \Delta_{\mu}(x) \psi(x),
$$

where

$$
\Delta_{\mu} \psi(x)=\frac{1}{2}\left(\mathcal{U}_{\mu}(x) \psi(x+\mu)-\mathcal{U}_{\mu}^{T}(x-\mu) \psi(x-\mu)\right),
$$

which reveals explicitly the antisymmetric character of the reduced fermion operator. This reduced action is invariant under two symmetries in addition to gauge invariance, a continuous $U(1)$ symmetry which acts on the fermions

$$
\psi(x) \rightarrow e^{i \alpha \epsilon(x)} \psi(x)
$$

and a discrete shift symmetry

$$
\psi \rightarrow \xi_{\rho} \psi(x+\rho),
$$

where $\xi_{\mu}=(-1)^{\sum_{i=\mu+1}^{d-1} x_{i}}$. Since for reduced fermions one keeps only $\psi$ or $\bar{\psi}$ at each site the usual staggered fermion mass term does not exist. However $\psi^{a} \psi^{b} \epsilon_{a b}$ is clearly a gauge invariant fermion bilinear for fermions transforming in the fundamental representation of $S U(2)$ and can hence be added to the fermion action, ${ }^{1}$

$$
\delta S=O_{S}=m \sum_{x} \epsilon(x) \psi^{a}(x) \psi^{b}(x) \epsilon_{a b} .
$$

To understand why the parity factor $\epsilon(x)$ appears in the mass term consider the full fermion operator

$$
D=\eta_{\mu}(x) \Delta_{\mu}^{a b}+m \epsilon(x) \epsilon^{a b} .
$$

The poles of the associated propagator are determined by the zeroes of $D^{2}$. Using the fact that the parity operator $\epsilon(x)$ anticommutes with the symmetric difference operator $\Delta_{\mu}$ allows one to write

$$
-D^{2}=-\Delta^{\mu} \Delta_{\mu}+m^{2}
$$

which exhibits the correct pole structure for a massive fermion (in Euclidean space). Notice that this mass operator induces the breaking $U(1) \rightarrow Z_{2}$. Alternatively, we can retain the $U(1)$ symmetry by adding a gauge invariant one link mass term which then breaks the shift symmetry.

$$
O_{L}=m_{1} \sum_{x, \mu} \frac{1}{2} \xi_{\mu}(x) \epsilon(x) \psi^{T}(x) \mathcal{M}_{\mu} \psi(x),
$$

where

$\mathcal{M}_{\mu} \psi(x)=\frac{1}{2}\left[\mathcal{U}_{\mu}(x) \psi^{b}(x+\mu)+\mathcal{U}_{\mu}^{T}(x-\mu) \psi(x-\mu)\right]$.

Notice the addition of $O_{S}$ and $O_{L}$ to the action preserves the antisymmetry of the fermion operator. In our numerical work we have investigated the effects of both of these mass terms. For a full staggered field the symmetry breaking patterns are a little different. For such a staggered field in a pseudoreal representation we can pair the $\psi$ and $\bar{\psi}$ at each site into a doublet with the kinetic operator now being invariant under a $U(2)$ symmetry. In this case a site mass term now breaks $U(2) \rightarrow O(2)$. Such a symmetry breaking pattern could also be obtained by using two reduced

\footnotetext{
${ }^{1}$ Notice the analog of this term vanishes for two continuum Weyl fermions because of an additional contraction over Lorentz indices unless the fermions carry additional flavor indices.
} 
staggered fields. Once we integrate the fermions we generate a Pfaffian. Since the fermion operator is antisymmetric its eigenvalues come in pairs $(\lambda,-\lambda)$. Additionally the pseudoreal nature of the representation implies that $U_{\mu}^{*}(x)=\sigma_{2} U_{\mu}(x) \sigma_{2}$, which ensures that every eigenvalue $\lambda_{n}$ (generically complex) and corresponding eigenvector $v_{n}$ is paired with another with eigenvalue $\lambda_{n}^{*}$ and eigenvector $\sigma_{2} v_{n}^{*}$. This quartic structure of the spectrum ensures that the Pfaffian is positive definite and can hence be written $P f(D)=\operatorname{det}\left(D^{\dagger} D\right)^{\frac{1}{4}}$, which is suitable for use in a Monte Carlo algorithm [13]. ${ }^{2}$ For the gauge part of the $S U(2)$ action we employ the standard Wilson action

$$
S_{G}=\sum_{x} \sum_{\mu<\nu}-\frac{\beta}{2 N} \operatorname{Tr}\left[U_{\mu \nu}(x)+U_{\mu \nu}^{\dagger}(x)\right] .
$$

The full action used for lattice simulation is given by

$$
S=S_{F}+S_{G}+O_{S}+O_{L}
$$

\section{NUMERICAL RESULTS}

We implemented the rational hybrid monte carlo algorithm to simulate the model exploring lattice volumes up to $16^{4}$ with gauge couplings spanning $\beta=0.5-4.0$ and for a wide range of site and link masses. Figures 1 and 2 show plots of the expectation values of the site and link bilinears for $m=m_{1}=0.1$ as a function of the gauge coupling $\beta$ for several lattice volumes. Both vacuum expectation values (vevs) are driven to small values for large $\beta$ as expected since the model enters a deconfined phase in that regime which can be seen clearly in Fig. 3 which shows the Polyakov line as a function of gauge coupling for $m=$ $m_{1}=0.1$ on a $6^{4}$ site lattice. The Polyakov line functions as a quasiorder parameter for the breaking of center symmetry and runs from small to large values as the system deconfines. However in a dynamical setup Polyakov line susceptibility does not show anything interesting for characterizing the order of transition. Of course the key question is whether one or more of these bilinears vev remains nonzero in the thermodynamic limit as the bare fermion mass is sent to zero. We focus on the largest values of the (inverse) gauge coupling (smallest lattice spacing) which clearly lie within the confining regime of the theory on the lattice volumes we have simulated. In Figs. 4 and 5 we show plots of the expectation values of the two blinears vs the bare fermion mass $m=m_{1}$ for gauge coupling $\beta=1.8$ for a range of lattice volumes. Clearly the link vev shows no strong volume dependence and smoothly goes to zero as the external mass is sent to zero. This is consistent with work by Follana et al. [3] for full staggered fermions in quenched approximation. The site bilinear shows a very

\footnotetext{
${ }^{2}$ An exception to this can occur if the fermion operator develops a purely real eigenvalue which is then unpaired. We have seen no sign of such eigenvalues in our simulations.
}

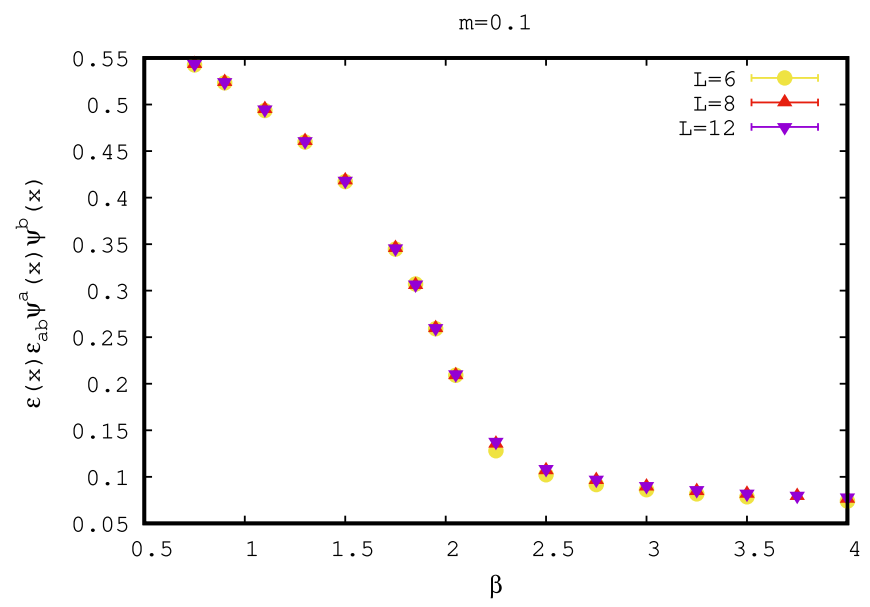

FIG. 1. $\left\langle O_{S}\right\rangle$ with $m=m_{1}=0.1$ for $L=6,8,12$.

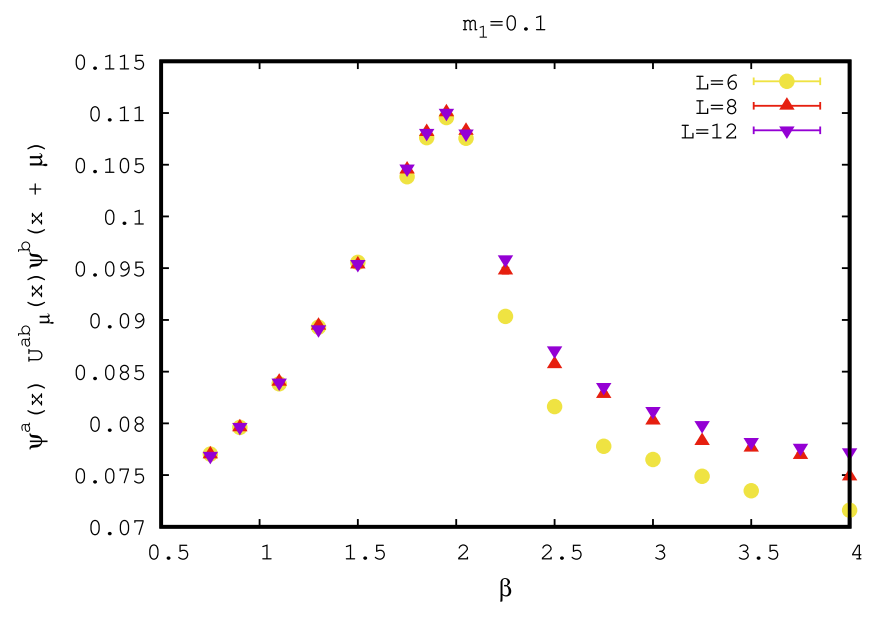

FIG. 2. $\left\langle O_{L}\right\rangle$ with $m=m_{1}=0.1$ for $L=6,8,12$.

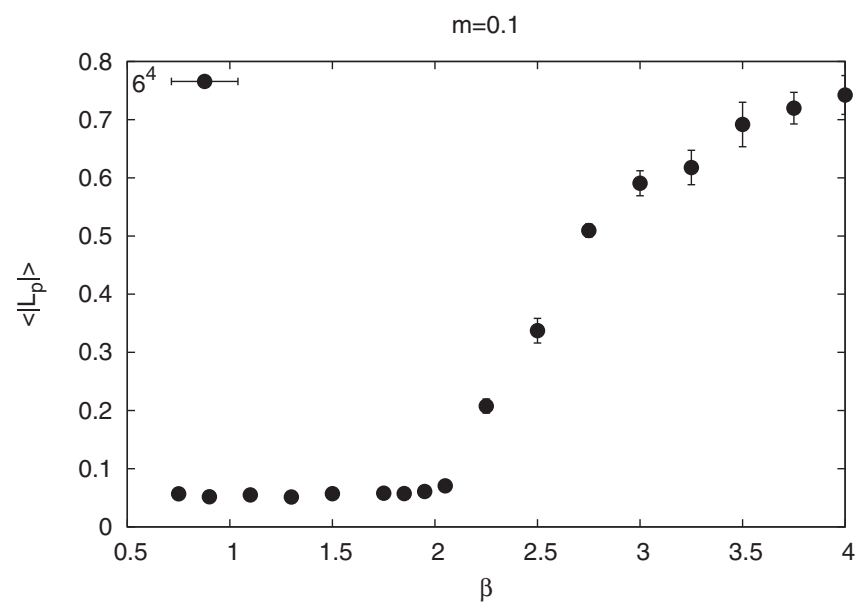

FIG. 3. $\left\langle\left|L_{p}(x)\right|\right\rangle$ Polyakov line for $L=6$ and $m=0.1$.

different behavior with the measured vev growing with volume at small mass. This is the behavior needed for a nonzero condensate to survive the zero mass limit, and indeed the data is quite consistent with the presence of a 


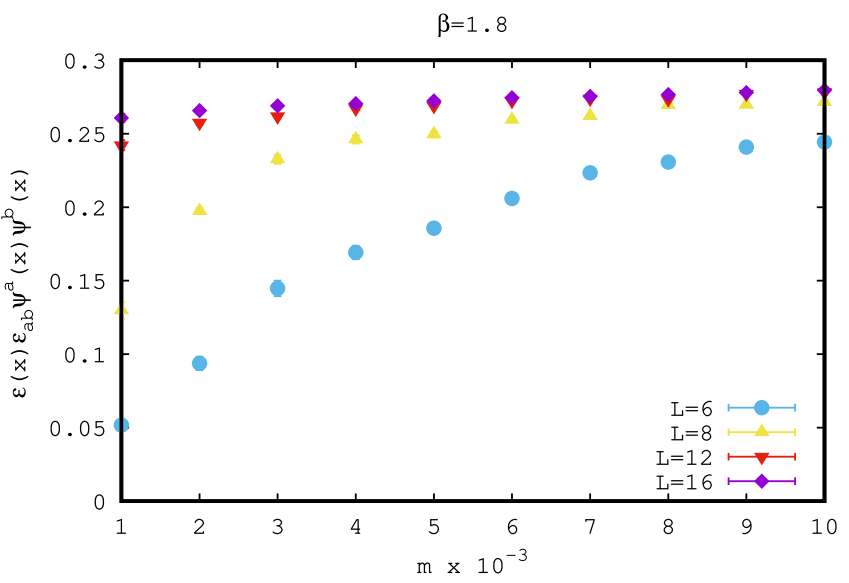

FIG. 4. $\left\langle O_{S}\right\rangle$ vs $m$ at $\beta=1.8$ for $L=6,8,12,16$.

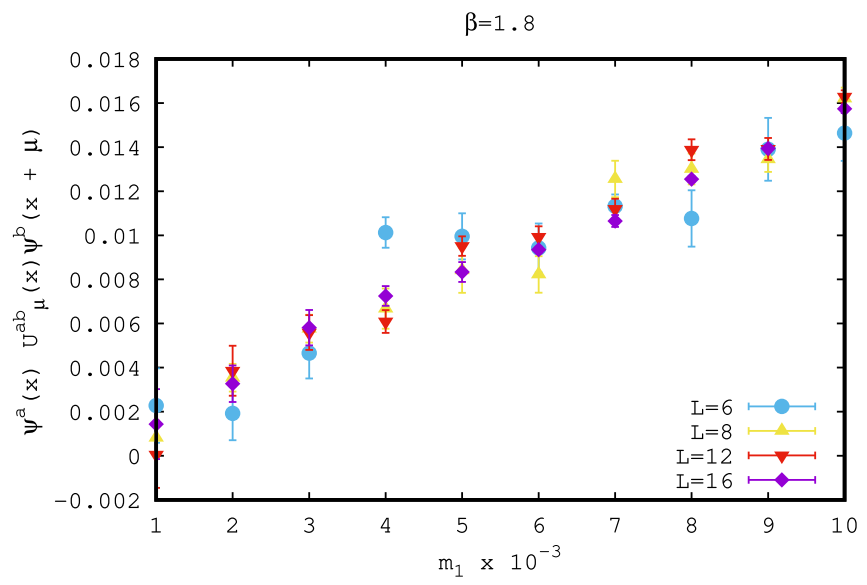

FIG. 5. $\left\langle O_{L}\right\rangle$ vs $m_{1}$ at $\beta=1.8$ for $L=6,8,12,16$.

nonzero site condensate in that limit. To gain confidence in this result we repeated the analysis for $\beta=1.7$ (Figs. 6 and 7) corresponding to a larger value of the lattice spacing. The overall conclusion remains the same, and we infer that the preferred breaking channel for the simple reduced staggered fermions studied here corresponds to $U(1) \rightarrow$ $Z_{2}{ }^{3}$ We can confirm these conclusions by looking for the corresponding Goldstone boson-the pion-whose correlator is given by

$$
\langle\phi(x) \phi(y)\rangle=\left\langle\epsilon^{a b} \psi^{a}(x) \psi^{b}(x) \epsilon^{c d} \psi^{c}(y) \psi^{d}(y)\right\rangle .
$$

A typical correlator is shown at $m=0.1$ and $\beta=1.8$ on a $8^{3} \times 32$ lattice in Fig. 8. We use the standard fit $C_{\pi}(t) \sim$ $A\left[\exp \left(-a m_{\pi}(t)+\exp \left(-a m_{\pi}(T-t)\right)\right]\right.$ to extract the pion mass. In Fig. 9 we plot the pion mass as a function of the bare quark mass $m$. For this calculation the smallest quark

\footnotetext{
${ }^{3}$ Similar results were observed by Follana [3] in the quenched approximation although the nature of the condensate changed when a smeared action was employed.
}

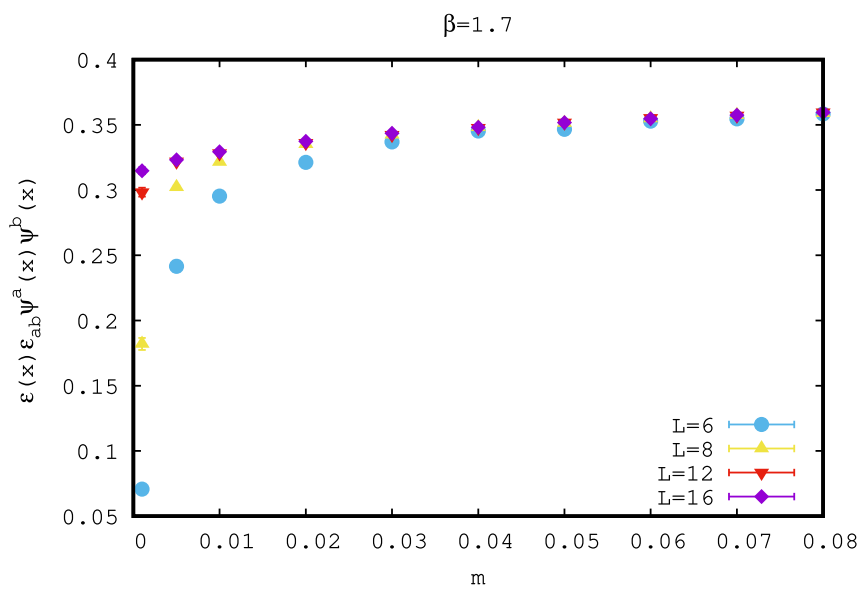

FIG. 6. $\left\langle O_{S}\right\rangle$ vs $m$ at $\beta=1.7$ for $L=6,8,12,16$.

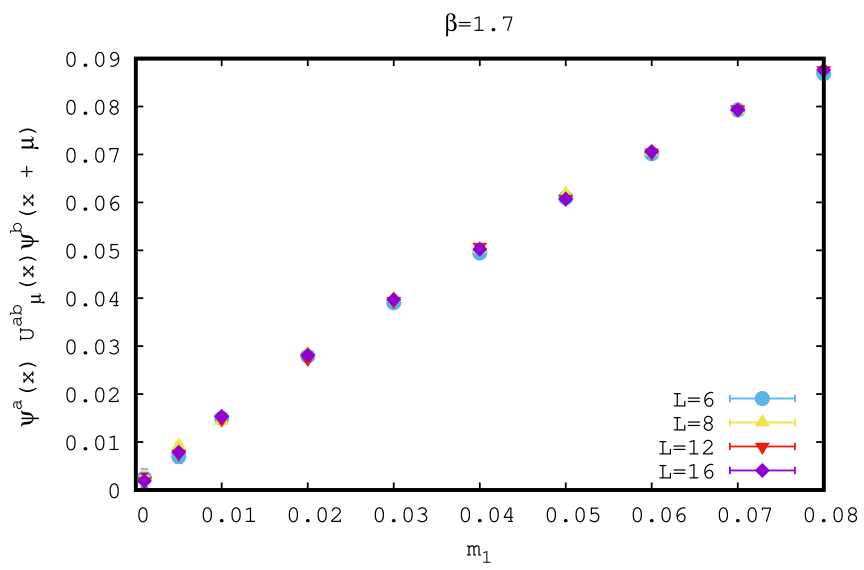

FIG. 7. $\left\langle O_{L}\right\rangle$ vs $m_{1}$ at $\beta=1.7$ for $L=6,8,12,16$.

mass we use is $m=0.01$ which ensures safe distance from epsilon regime where finite volume effects drive the condensate to zero in $m \rightarrow 0$ limit. The solid line is a fit to the expected square root form and corresponds to the

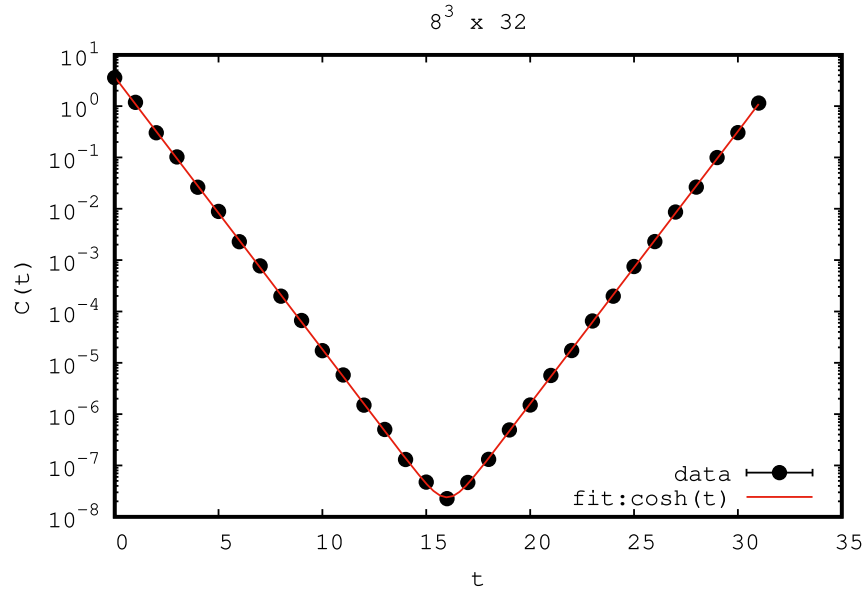

FIG. 8. $\langle C(t)\rangle$ :pion correlator with quark mass $m=0.2$ for $8^{3} \times 32$. 


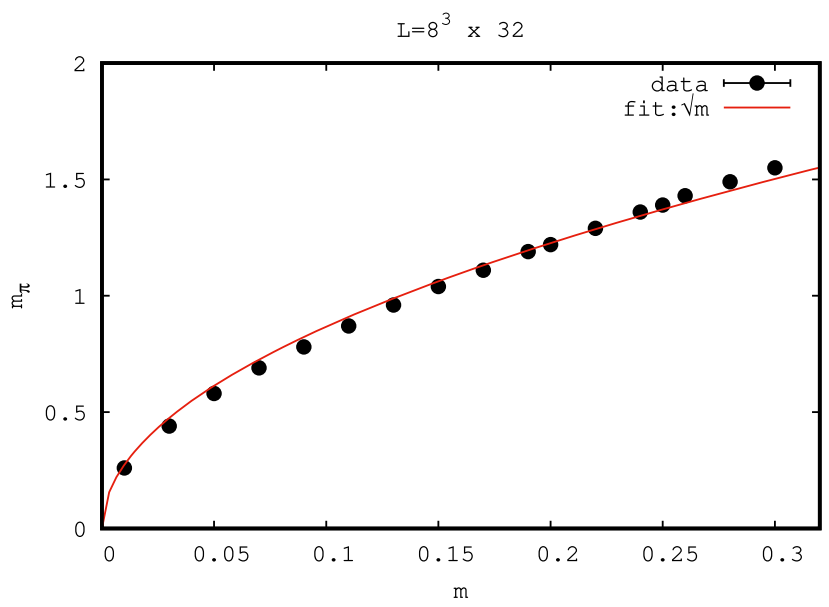

FIG. 9. $m_{\pi}$ vs $m$ :Pion mass vs bare quark mass for $8^{3} \times 32$.

standard GMOR prediction confirming that this state is indeed a pion resulting from spontaneous breaking of the $U(1)$ symmetry.

\section{SUMMARY}

In this paper we perform the first studies of $S U(2)$ lattice gauge theory with dynamical reduced staggered fermions. The pseudoreal nature of the fundamental representation of $S U(2)$ allows us to employ the standard rational hybrid monte carlo algorithm without encountering a sign problem. Unlike $S U(N)$ for $N>2$ a gauge invariant site mass term is allowed, and we investigate the model including both this term and a gauge invariant one-link mass operator. We find strong evidence that a site bilinear fermion condensate is formed at strong coupling spontaneously breaking an exact $U(1)$ symmetry down to $Z_{2}$. We find good evidence for the corresponding Goldstone bosonthe pion. These results are consistent with previous studies that used the spectrum of low-lying eigenmodes of the quenched Dirac operator to find evidence for chiral symmetry breaking in this theory. Our results strengthen these conclusions and support the analysis given in [5]. This work is motivated by an attempt to understand some of the novel phase structure in a related Higgs-Yukawa model involving reduced staggered fermions interacting with $S U(2)$ gauge fields. The current work establishes the bedrock for understanding the results of those studies the results of which will be reported soon.

\section{ACKNOWLEDGMENTS}

This work is supported in part by the U.S. Department of Energy, Office of Science, Office of High Energy Physics, under Award No. DE-SC0009998. Numerical computations were performed at Fermilab using USQCD resources.
[1] S. Aoki et al., Review of lattice results concerning lowenergy particle physics, Eur. Phys. J. C 77, 112 (2017).

[2] C. van den Doel and J. Smit, Dynamical symmetry breaking in two flavor $\mathrm{SU}(N)$ and $\mathrm{SO}(N)$ lattice gauge theories, Nucl. Phys. B228, 122 (1983).

[3] E. Follana, C. T. H. Davies, and A. Hart, Improved staggered eigenvalues and epsilon regime universality in $\mathrm{SU}(2)$, Proc. Sci. LAT2006 (2006) 051.

[4] S. Catterall and A. Veernala, Four fermion interactions in non-Abelian gauge theory, Phys. Rev. D 87, 114507 (2013).

[5] P. H. Damgaard, U. M. Heller, R. Niclasen, and B. Svetitsky, Patterns of spontaneous chiral symmetry breaking in vectorlike gauge theories, Nucl. Phys. B633, 97 (2002).

[6] V. Ayyar and S. Chandrasekharan, Massive fermions without fermion bilinear condensates, Phys. Rev. D 91, 065035 (2015).

[7] S. Catterall, Fermion mass without symmetry breaking, J. High Energy Phys. 01 (2016) 121.
[8] Y.-Z. You, Y.-C. He, C. Xu, and A. Vishwanath, Symmetric Fermion Mass Generation as Deconfined Quantum Criticality, Phys. Rev. X 8, 011026 (2018).

[9] V. Ayyar and S. Chandrasekharan, Fermion masses through four-fermion condensates, J. High Energy Phys. 10 (2016) 058.

[10] S. Catterall and D. Schaich, Novel phases in strongly coupled four-fermion theories, Phys. Rev. D 96, 034506 (2017).

[11] S. Catterall and N. Butt, $\mathrm{SO}(4)$ invariant Higgs-Yukawa model with reduced staggered fermions, Phys. Rev. D 98, 114514 (2018).

[12] S. Catterall and N. Butt, Topology and strong four fermion interactions in four dimensions, Phys. Rev. D 97, 094502 (2018).

[13] M. A. Clark and A. D. Kennedy, The RHMC algorithm for two flavors of dynamical staggered fermions, Nucl. Phys. B, Proc. Suppl. 129, 850 (2004). 